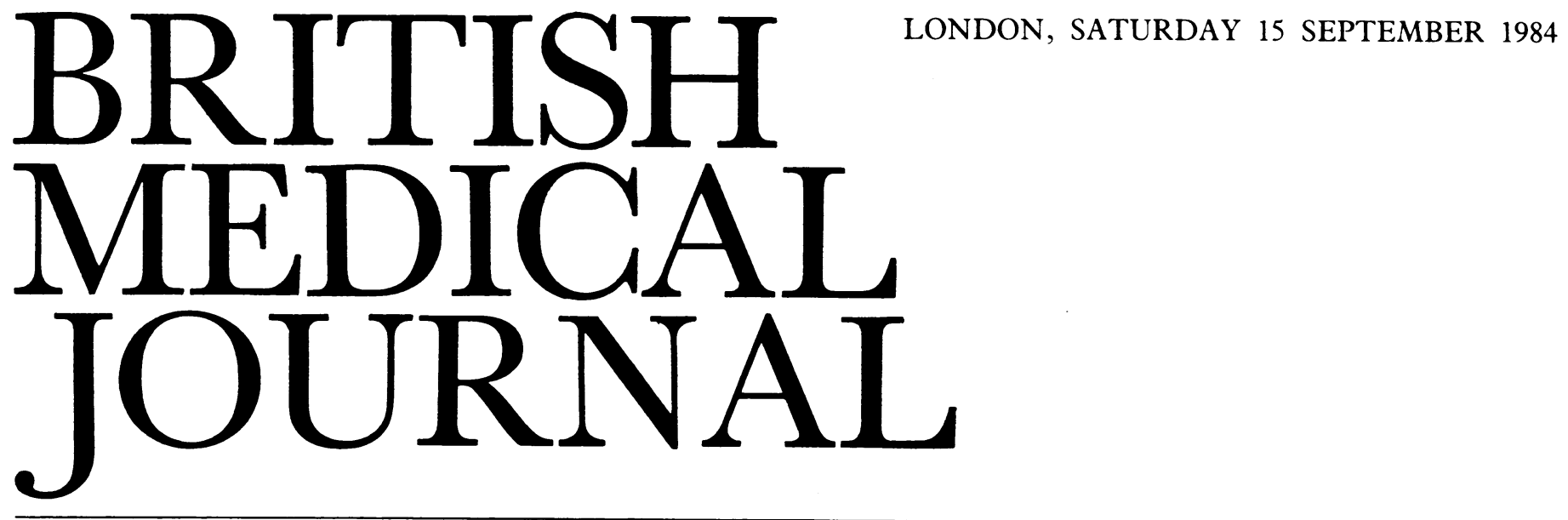

Of the several papers published on unrecognised psychiatric illness in medical patients, most have appeared in psychiatric journals and may not have come to the attention of general or specialist physicians. Nevertheless, in the Lancet review in 1979 the incidence of psychiatric illness reported ranged from $25 \%$ to $83 \% .^{1}$ Clearly this figure depended on how psychiatric illness was defined and the type of hospital surveyed. Many of the papers reviewed had been by psychiatrists, who pointed to the failure of their general medical colleagues to recognise psychiatric problems in their patients. This judgment, however, was largely based on a scrutiny of the case notes, summaries, and doctors' letters to decide whether the psychiatric aspect had been recognised, and it may have underestimated the ability of the non-psychiatrist to spot a psychiatric component in a patient's illness. To make a balanced assessment three problems need to be considered. Firstly, how common is psychiatric illness in medical patients? Secondly, how important is it that it should be recognised and documented? Thirdly, if it is important what can be done to ensure more frequent recognition?

In a study in the particularly busy medical wards of the Radcliffe Infirmary in Oxford, 170 patients were given the general health questionnaire,,$^{2}$ and the 77 who had a score suggesting psychiatric problems were interviewed by a psychiatrist." (It is worth noting that of the 93 patients thought to be psychiatrically normal, six were later found to have problems.) As a result of the interview 20 patients were judged to have moderate or severe psychiatric problems and 25 mild disturbances. Of these 45 patients, 22 had been recognised by the medical staff as having psychiatric problems. Twenty five were depressed, 10 had anxiety, and one phobias. Physicians were most likely to recognise psychiatric disturbances if the patient was uncooperative or noisy, weepy or agitated, or if a psychiatric history was mentioned in the general practitioner's referral letter. Quiet and uncomplaining patients tended not to be recognised or referred. In a recent study of 100 patients in the nourological wards of a Manchester teaching hospital (p 656) the incidence of psychiatric disorder was assessed, by a psychiatrist, as about $40 \%$, with only just over a quarter of the cases being recognised by the neurologists. In this series also depression and anxiety were the commonest diagnoses.

Clearly, an appreciable amount of anxiety and depression does go unrecognised in our general medical and neurological wards, but would it help if it were recognised and appropriate advice was sought? The patients in the Oxford study have now been followed up, and in many cases the psychiatric problems persisted, particularly when there was a history of psychiatric trouble before the acute admission. ${ }^{4}$ This suggests that detection is worth while so that persistent problems may be expertly assessed and treated if necessary. A study from the Johns Hopkins Hospital found that about a quarter of the 150 inpatients in the acute medical wards were depressed, and the authors compared the characteristics of these depressed patients with those in a series of depressed patients in the psychiatric wards. ${ }^{5}$ The average age of those in the medical wards was higher (51.6 $v 35.8$ years) and their depression not so severe, with fewer showing suicidal tendencies. In addition, the medical patients showed more anxiety, hopelessness, and helplessness, although many improved spontaneously as their illnesses resolved. Two earlier papers from Florida recorded depression in 31 out of 153 patients and certain points about uses and abuses of psychiatric consultation were emphasised. ${ }^{67}$ They concluded that the physician or surgeon should not delay seeking help until the situation had become critical and that when asked to come the psychiatrist should respond promptly. This is essential in wards which have a rapid turnover of patients. Another important point is that it is both courteous and prudent to speak to the patient's general practitioner before psychiatric referral is made.

If we accept that patients with psychological problems would benefit from psychiatric help, how can we ensure better recognition of these problems in the general wards? It has been suggested that a simple questionnaire (general health questionnaire) should be given to patients who are not too ill and that those with a poor score should then have a psychiatric interview. ${ }^{1}$ This may seem a somewhat mechanistic approach, but it is worthy of further evaluation. If it is to be left to history taking by the ward staff we have the problem that in our hospitals this is done initially by the house officer, then by the registrar, and by any medical students and perhaps the nursing staff. On some wards the consultant will go over the story yet again. Such zealous history taking will not necessarily increase the rate of detection of psychiatric problems, and in a study comparing the ability of house officers, students, and nurses to recognise 
such problems the students were top ( $23 \%$ failures), house officers second (35\%), and, surprisingly, nurses bottom $(70 \%) .{ }^{8}$ Bridges and Goldberg in their paper in this week's $B M \mathcal{F}$ speak of neurologists, but it is not clear whether they are referring to the consultants or to the registrars, and it will be impossible to decide who recognised or failed to recognise a psychiatric component in the patient's illness.

In most hospitals, however, the most sensitive and comprehensive history is probably that taken by the registrar or his equivalent. How good it will be will depend on his experience and particular interest. An even better history would probably be obtained by the consultantprovided that he had adequate time to do this. Unfortunately, he seldom has-unlike the consultant physician or neurologist in private practice, who will set aside an hour to take a full history, examine the patient, and explain to him or her how he plans to proceed. This will be done in private, undisturbed by bleeps or other interruptions. If such an approach were possible with health service patients, and it should be, the psychiatric component of acute medical illnesses would be recognised more frequently. Expenditure on the National Health Service in Britain is less than in other developed countries. We do not have enough consultants in the acute medical and surgical specialties to allow them to devote sufficient time to each patient. Doctors in training rarely have sufficient time and many lack the experience to recognise psychiatric problems in their patients. Furthermore, facilities for a private, unhurried, and undisturbed interview are seldom available. The fact that the service is as good as it is is a tribute to the dedication of those who work in it. If the country could afford it, it could be very much better.

London W1

JOHN NABARRO

1 Anonymous. Psychiatric illness among medical patients [Editorial]. Lancet 1979;i:478-9. 1972. (Maudsley Monograph No 21.) 1972. (Maudsley Monograph No 21.)
general medical wards. Br Med $\mathcal{F}$ 1974; $1: 268-70$.

Hawton $\mathrm{KE}$. The long term outcome of psychiatric morbidity detected in general medical wards. f Psychosom Res 1981;25:237.43.

5 Moffic HS, Paykell ES. Depression in medical inpatients. Br $\mathcal{F}$ Psychiatry 1975:126:346-53. Schwab JJ, Bialow M, Brown JM, Holzer CE. Diagnosing depression in medical inpatients. Ann Intern Med 1967;67:695-707.

7 Schwab JJ, Brown JM. Uses and abuses of psychiatric consultation. FAMA 1968;205:65-8.

Knights EB, Folstein MF. Unsuspected emotional and cognitive disturbance in medical patients. Ann Intern Med 1977;87:723-4.

\section{Postmyocardial infarction syndrome}

In the postmyocardial infarction syndrome of Dressler, fever and pleuropericardial pain occur after a coronary occlusion. ${ }^{1}$ In nearly all respects the syndrome is similar to the postcardiotomy syndrome which affects patients recovering from heart surgery. ${ }^{2}$ A pericardial friction rub may be heard in most patients, and pericardial and pleural effusions are common. The syndrome is rarely seen earlier than the second week after myocardial infarction and its peak incidence is during the first three months. ${ }^{3}$ This is a self limiting condition: the symptoms do not last longer than four to six weeks, but they tend to recur-sometimes as late as two years after the initial episode. ${ }^{4}$

Careful and prolonged follow up of many patients is required to determine the incidence of the postmyocardial infarction syndrome. The logistical problem is compounded by the absence of a specific diagnostic marker. The largest study of this type was by Welin et al in 1809 patients with recent myocardial infarction. ${ }^{5}$ At least two features of postmyocardial infarction syndrome-pleuropericardial pain, fever, or high erythrocyte sedimentation rate-were fulfilled by $3 \cdot 3 \%$ of the patients during 12 months of follow up, a figure closely agreeing with the estimated 3-4\% incidence originally reported by Dressler. ${ }^{3}$ The factors which predispose towards development of the syndrome are not well defined. Welin et al found an association with large, complicated infarcts, 5 which might itself account for previously noted associations with ventricular aneurysm ${ }^{6}$ and simple postinfarction pericarditis. ${ }^{7}$ Not all investigators, however, have confirmed these observations, and cautious interpretation is necessary. ${ }^{38}$ It is not clear, for example, whether ventricular aneurysm represents a true predisposing factor or whether it reflects an adverse effect of corticosteroids used to treat established postmyocardial infarction syndrome. ${ }^{9}$

There are no simple diagnostic laboratory tests for the syndrome, though the erythrocyte sedimentation rate and the peripheral white cell count are usually raised. Specific serological abnormalities have been sought, based on the widely held view that the syndrome is a hypersensitivity reaction to autologous heart tissue with altered antigenicity as a result of ischaemia. Viral infection and blood in the pericardium have also been invoked as possible causes of the hypersensitivity. ${ }^{10}$ Certainly the characteristic latency period, the polyserositis, and the favourable response to immunosuppressive drugs are circumstantial support for an autoimmune aetiology. Heart reactive antibodies are frequently detected after myocardial infarction but the finding is nonspecific (particularly with a weakly positive result) and does not necessarily herald the development of the postmyocardial infarction syndrome. ${ }^{11-15}$ On the other hand, a strongly positive test result appears to be more specific, ${ }^{13} 15$ and Williams et al have proposed this as a potentially useful diagnostic marker. ${ }^{15}$ Nevertheless, such serological testing requires technical and methodological skills that are likely to confine its application to specialist centres. ${ }^{16} 17$

The diagnostic criteria chosen by Welin et al are satisfactory for most cases of postmyocardial infarction syndrome. ${ }^{5}$ Radiographic and ultrasound studies provide additional information on the presence of pleural and pericardial effusions. Further investigation is necessary, however, to exclude the possibility of extension or recurrence of myocardial infarction, pulmonary embolism, and congestive heart failure, all of which are frequent in the weeks after infarction, and all of which have a poor prognosis. By contrast, the postmyocardial infarction syndrome is usually a benign illness which does not affect cumulative mortality. ${ }^{5}$ The only potentially lethal complication is cardiac tamponade, ${ }^{18}$ which is rare and tends to occur in patients having anticoagulant treatment, who are at risk of pericardial haemorrhage. ${ }^{3}$ Anticoagulants should not, therefore, be prescribed for patients with this syndrome.

Evaluation of treatment is difficult because the postmyocardial infarction syndrome is self limiting. Simple antiinflammatory analgesics such as aspirin will usually control symptoms but do not appear to influence the course of the illness. In severe cases treatment with corticosteroids often results in a dramatic response with resolution of fever and relief of pain within 24 hours, although the condition tends to relapse after stopping treatment. ${ }^{4}$ Whether this is a specific adverse effect of treatment or a characteristic of the illness is 